\title{
Surfaces with surjective endomorphisms of any given degree
}

\section{Antonio Rapagnetta \& Pietro Sabatino}

Rendiconti del Circolo Matematico di Palermo

ISSN 0009-725X

Rend. Circ. Mat. Palermo

DOI 10.1007/s12215-011-0068-9

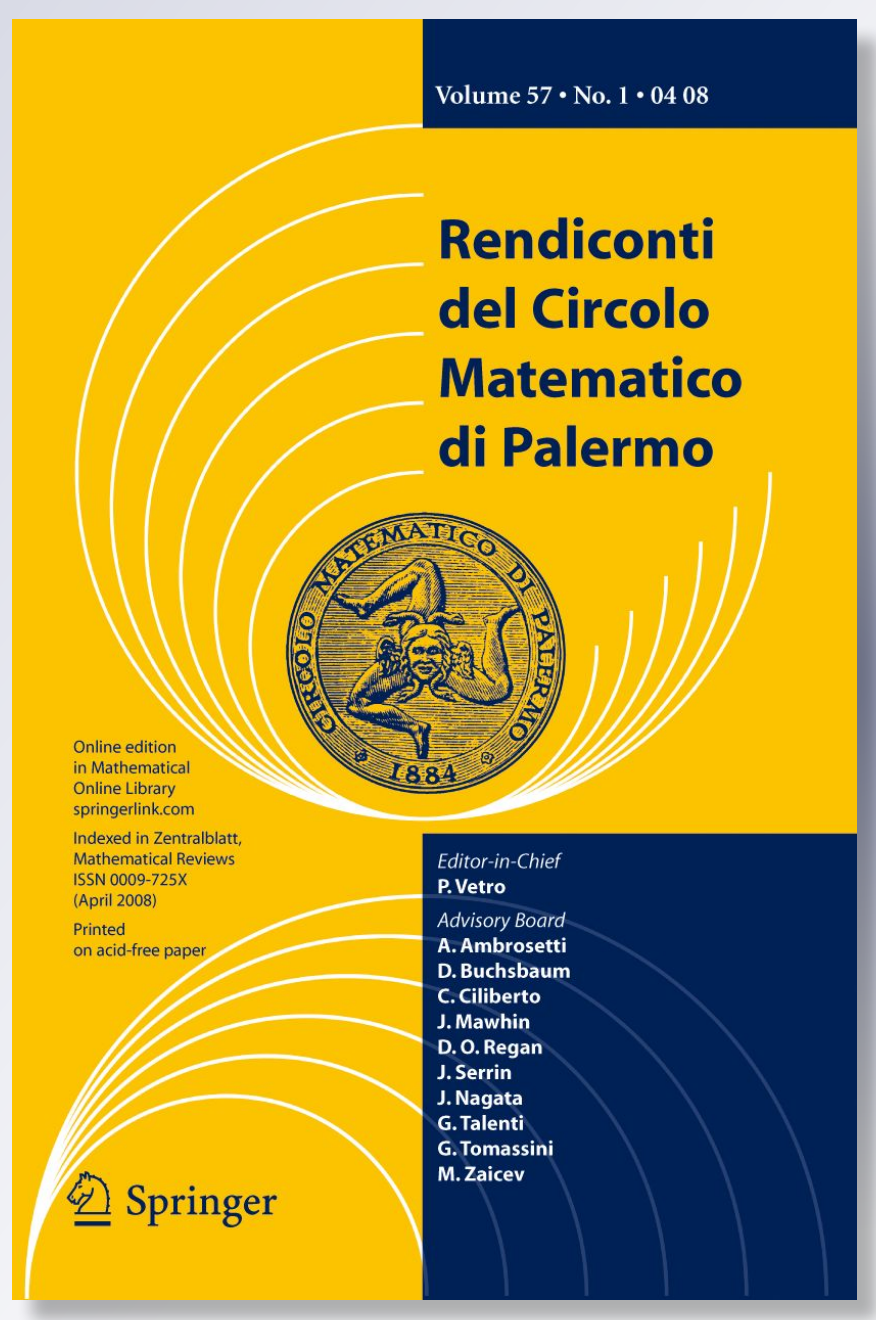

Springer 
Your article is protected by copyright and all rights are held exclusively by SpringerVerlag. This e-offprint is for personal use only and shall not be self-archived in electronic repositories. If you wish to self-archive your work, please use the accepted author's version for posting to your own website or your institution's repository. You may further deposit the accepted author's version on a funder's repository at a funder's request, provided it is not made publicly available until 12 months after publication. 


\title{
Surfaces with surjective endomorphisms of any given degree
}

\author{
Antonio Rapagnetta $\cdot$ Pietro Sabatino
}

Received: 21 December 2010 / Accepted: 8 July 2011

(C) Springer-Verlag 2011

\begin{abstract}
We present a complete classification of complex projective surfaces $X$ with nontrivial self-maps (i.e. surjective morphisms $f: X \rightarrow X$ which are not isomorphisms) of any given degree. Our starting point are results contained in Fujimoto (Publ. Res. Inst. Math. Sci. 38(1):33-92, 2005) and Nakayama (Kyushu J. Math. 56(2):433-446, 2002), they provide a list of surfaces that admit at least one nontrivial self-map. By a case by case analysis that blends geometrical and arithmetical arguments, we then exclude that certain prime numbers appear as degrees of nontrivial self-maps of certain surfaces.
\end{abstract}

Keywords Nontrivial surjective endomorphism · Projective bundles · Étale quotients

Mathematics Subject Classification (2010) Primary 14J25 - Secondary 14J26 · 14A10

\section{Introduction and statement of the result}

We work over the complex field $\mathbb{C}$. All the varieties that we will consider will be projective and smooth, in case of exceptions we will explicitly state it.

Definition 1 Let $X$ be a complex projective variety and let $f: X \rightarrow X$ be a surjective endomorphism of $X$, i.e. a morphism of $X$ onto itself. $f$ is said to be a nontrivial self-map if it is not an isomorphism, or equivalently if the degree of $f$ is greater than or equal to 2 .

In what follows we will provide a complete classification of surfaces that admit a nontrivial self-map of any given degree, this is the content of the following theorem.

\footnotetext{
A. Rapagnetta

Via Della Ricerca Scientifica 1, 00133 Rome, Italy

e-mail: rapagnet@axp.mat.uniroma2.it

P. Sabatino $(\bowtie)$

Via Delle Mimose 9 Int. 14, 00172 Rome, Italy

e-mail: pietrsabat@gmail.com
} 
Theorem 2 A surface admits self-maps of any given degree if and only if it is one of the following:

(i) $\mathbb{P}_{1} \times \mathbb{P}_{1}$

(ii) Étale quotient of $\mathbb{P}^{1} \times C, C$ a smooth curve with $g(C) \geq 2$, by a cyclic group $G$ of automorphisms of $C$ acting freely on $C$ and faithfully on $\mathbb{P}^{1}$. If $G$ is not trivial then its order is a prime $p$ and for every $\delta \in(\mathbb{Z} / p \mathbb{Z})^{*}$ there exists $\varphi \in \operatorname{Aut}(C)$ such that $\varphi \circ g=g^{ \pm \delta} \circ \varphi$ for every $g \in G$.

(iii) $X$ is $\mathbb{P}^{1}$-bundle over an elliptic curve $E, \mathbb{P}\left(\mathcal{O}_{E} \oplus \mathcal{L}\right)$, where $\mathcal{L}$ is a $k$-torsion line bundle on $E$ and either $k=1,2,3$ or

(iiia) $k=4, E$ is the elliptic curve relative to the lattice $\left\langle 1, \frac{1}{2}+\frac{i \sqrt{7}}{2}\right\rangle$, and $\mu^{*} \mathcal{L}=0$ where $\mu$ is the endomorphism of $E$ induced by multiplication by either $\frac{3}{2}+i \frac{\sqrt{7}}{2}$ or $\frac{3}{2}-i \frac{\sqrt{7}}{2}$.

(iiib) $k=5, E$ is the elliptic curve relative to the lattice $\langle 1, i\rangle$, and $\mu^{*} \mathcal{L}=0$ where $\mu$ is the endomorphism of $E$ induced by multiplication by either $2+i$ or $2-i$.

(iiic) $k=7, E$ is the elliptic curve relative to the lattice $\left\langle 1, \frac{1}{2}+\frac{i \sqrt{3}}{2}\right\rangle$, and $\mu^{*} \mathcal{L}=0$ where $\mu$ is the endomorphism of $E$ induced by multiplication by either $\frac{5}{2}+\frac{i \sqrt{3}}{2}$ or $\frac{5}{2}-\frac{i \sqrt{3}}{2}$.

A couple of remarks about the statement of Theorem 2 are in order. Regarding items (iiia)-(iiic), recall that there is a one-to-one correspondence between endomorphisms of a given elliptic curve $E$ and complex numbers $\alpha$ such that $\alpha \cdot \Lambda \subseteq \Lambda$, where $\Lambda$ denotes the lattice relative to the curve $E$ (see Remark 28). Moreover, a priori, it is not clear whether or not there exist examples of surfaces that satisfies Theorem 2(ii). But, it turns out that such examples exist and we describe some of them in Example 21. Our description is based on a classical result of Hurwitz, according to which every finite group can be realized as an automorphism group of some compact Riemann surface (see [5] for instance).

The starting point of our analysis is the following result, it provides a list of surfaces that do admit at least one nontrivial self-map.

Theorem 3 Let $X$ be a complex projective surface, $X$ admits a nontrivial self-map if and only if one of the following conditions is satisfied

(i) $X$ is an Abelian surface;

(ii) $X$ is an hyperelliptic surface, $X$ is an entry in the list of Bagnera-de Franchis (see for example [2, pp. 83-84]);

(iii) $X$ is a minimal surface with $\kappa(X)=1$ and $\chi\left(\mathcal{O}_{X}\right)=0$;

(iv) $X$ is a toric surface;

(v) $X$ is a $\mathbb{P}^{1}$-bundle over an elliptic curve;

(vi) $X$ is a $\mathbb{P}^{1}$-bundle over a nonsingular projective curve $B$ with $g(B)>1$ such that $X \times_{B}$ $B^{\prime}$ is trivial after an étale base change $B^{\prime} \rightarrow B$.

Proof See [6, Theorem 3.2] for the case $\kappa(X) \geq 0$ and [8, Theorem 3] for the case $\kappa(X)=-\infty$.

Since the degree of morphisms of $X$ onto itself is multiplicative with respect to the composition of maps, a surface admits nontrivial self-maps of any given degree if and only if it admits nontrivial self-maps of any given prime degree. Hence trough the rest of this paper we will restrict our analysis to prime degrees without any further comment. Theorem 2 will 
follow from Theorem 3 after a case by case analysis involving both geometric and arithmetic arguments.

Notations 4 We will denote by $\equiv_{\text {num }}$, $\equiv_{\text {lin }}$, respectively linear equivalence and numerical equivalence of divisors. For a locally free sheaf $\mathcal{E}$ on a smooth projective variety we put $\mathbb{P}(\mathcal{E}):=\operatorname{Proj}\left(\operatorname{Sym}\left(\mathcal{E}^{\vee}\right)\right)$, note that our notation coincide with the $\mathbb{P}\left(\mathcal{E}^{\vee}\right)$ of Hartshorne's book.

\section{Abelian surfaces and the case $\kappa(X) \geq 0$}

First of all we are going to analyse case (i) of Theorem 3, namely abelian surfaces. This case will be a direct consequence of the lemma below.

Lemma 5 Let $T$ be a complex torus. There exist an infinite number of primes that do not appear as degree of a nontrivial self-map of $T$.

Proof Let $V$ be a complex vector space of dimension $g$ and $\Lambda$ a lattice in $V$. Put $T=$ $V / \Lambda$. Every nontrivial self-map $f: T \rightarrow T$ is the composition of a translation and a group endomorphism of $T$, then we may suppose, without loss of generality, that $f$ is a group endomorphism of $T$. Denote by $\operatorname{End}(T)$ the set of group endomorphisms of $T$. Denote by $\rho_{a}$ and $\rho_{r}$ extensions of the analytic and rational representation of $\operatorname{End}(T)$ to $\operatorname{End}_{\mathbb{Q}}(T)=$ $\operatorname{End}(T) \otimes \mathbb{Q}$ (see $[4$, p. 10]). The extended rational representation

$$
\rho_{r} \otimes 1: \operatorname{End}_{\mathbb{Q}}(T) \otimes \mathbb{C} \rightarrow \operatorname{End}_{\mathbb{C}}(\Lambda \otimes \mathbb{C}) \simeq \operatorname{End}_{\mathbb{C}}(V \times V)
$$

is equivalent to the direct sum of the analytic representation and its conjugate [4, Proposition 1.2.3]

$$
\rho_{r} \otimes 1 \simeq \rho_{a} \oplus \overline{\rho_{a}} .
$$

Observe now that $\operatorname{deg}(f)=\operatorname{det} \rho_{r}(f)=\operatorname{det} \rho_{a}(f) \overline{\operatorname{det} \rho_{a}(f)}$. Since $\rho_{r}(f)$ has integer entries its eigenvalues are all algebraic integers, it follows by (1) that det $\rho_{a}(f)$ is also an algebraic integer. Moreover $\operatorname{det} \rho_{a}(f)$ for all $f \in \operatorname{End}(T)$, are all contained in the same number field that depends only on $T$. Indeed they generate an extension, say $K$, contained in a finitely generated extension of $\mathbb{Q}$, namely the extension generated by the entries of a period matrix for $T$. It is a well known fact that $K$ is finitely generated too [7, p. 229, Remark]. We may moreover suppose, without loss of generality, that $K$ is Galois over $\mathbb{Q}$. Summing up we have that if a prime $p$ appears as the degree of an endomorphism of $T$ then

$$
p=\alpha \bar{\alpha}, \quad \alpha \in K
$$

$K$ a Galois number field and $\alpha$ an algebraic integer. Moreover in what follows we may and will restrict our attention to primes that do not ramify in the extension $K$, since the number of ramified primes is finite. If a prime satisfies (2) then no prime ideal in $K$ that divides $(p)$, the ideal generated by $p$ in the ring of integers of $K$, admits complex conjugation as its Frobenius. It follows by Čebotarev density theorem [9, Theorem 13.4, p. 545] that the complementary set of the set of primes that satisfy (2) has analytic density strictly greater than zero. 
Corollary 6 Let $A$ be an abelian variety, then there are an infinite number of primes that do not appear as degree of a nontrivial self-map of A.

Remark 7 Riemann-Hurwitz formula implies that if a curve $C$ possesses a nontrivial selfmap then $g(C) \leq 1$. Moreover by Corollary 6 it follows that $\mathbb{P}^{1}$ is the only curve with nontrivial self-maps of any given degree.

Now we come to (ii) and (iii) of Theorem 3. We start the proof of the following proposition considering surfaces of Kodaira dimension one. After a preliminary argument we are left with surfaces such that $p_{g}=0$ and $q=1$. We are then able to treat them by an argument that holds for surfaces in (ii) of Theorem 3 too.

Proposition 8 Let $X$ be either a minimal surface with $\kappa(X)=1$ and $\chi\left(\mathcal{O}_{X}\right)=0$ or an hyperelliptic surface. $X$ fails to admit a nontrivial self-map of degree a given prime for an infinite number of primes.

Proof First of all suppose that $X$ is minimal, $\kappa(X)=1$ and $\chi\left(\mathcal{O}_{B}\right)=0$. Note that $K_{X}^{2}=0$ and then the topological Euler-Poincaré characteristic $e(X)$ is zero too. Moreover $X$ admits an elliptic fibration $\pi: X \rightarrow B, B$ a smooth curve, and since $e(X)=0$ singular fibres are multiples of a smooth elliptic curve [1, Proposition (11.4) and Remark (11.5), p. 118].

Arguing as in [3, Chap. VI] it follows that $X \simeq(F \times \widetilde{B}) / G$, where $F$ and $\widetilde{B}$ are smooth curves $g(F)=1, g(\widetilde{B}) \geq 2$ and $G$ is a group of automorphisms of $F$ and $\widetilde{B}$ such that $G$ acts freely on $F \times \widetilde{B}$. Moreover $\widetilde{B} / G \simeq B$ and

$$
\pi: X \simeq(F \times \widetilde{B}) / G \rightarrow B \simeq \widetilde{B} / G
$$

is the map induced by the projection of $F \times \widetilde{B}$ onto $\widetilde{B}$, in particular every smooth fiber of $\pi$ is an elliptic curve isomorphic to $F$.

Observe now that a suitable pluricanonical map factorizes through $\pi$ and an embedding of $B$ in some projective space [2, Proposition IX.3, p. 108]. Let

$$
f: X \rightarrow X
$$

be a nontrivial self-map. Pulling back multiples of canonical divisors by $f$ induces a map $f_{B}: B \rightarrow B$ such that the following diagram commutes

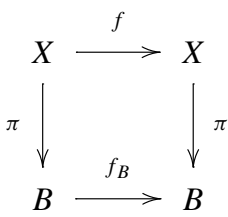

It follows that $\operatorname{deg} f=\operatorname{deg}\left(f_{B}\right) \operatorname{deg}\left(f_{\mid F}\right)$, where $\operatorname{deg}\left(f_{\mid F}\right)$ denotes the degree of the restriction of $f$ to a smooth fiber.

If $g(B)>1$ then $\operatorname{deg}\left(f_{B}\right)=1$ and $X$ fails to admit a nontrivial self-map of degree a given prime for an infinite number of primes, indeed the same holds for $F$ (Remark 7). If $B$ is an elliptic curve, then any prime appearing as the degree of an endomorphism of $X$ is also the degree of an endomorphism of the abelian surface $B \times F$, the missing primes are then infinite by Corollary 6. 
If $B$ is rational then

$$
\mathrm{H}^{1,0}(S) \cong \mathrm{H}^{1,0}(F \times \widetilde{B})^{G} \cong \mathrm{H}^{1,0}(F)^{G} \oplus \mathrm{H}^{1,0}(\widetilde{B})^{G} \cong \mathrm{H}^{1,0}(F / G) \oplus \mathrm{H}^{1,0}(B) .
$$

Since $\chi\left(\mathcal{O}_{X}\right)=0$ we have $q=1$ and $F / G$ is elliptic. The fibres of the natural map $\alpha:(F \times \widetilde{B}) \rightarrow F / G$ are connected and isomorphic to $\widetilde{B}$, it follows that $\alpha$ is the Albanese map of $X$. By the universal property of the Albanese map there exists a $\varphi: F / G \rightarrow F / G$ such that $\alpha \circ f=\varphi \circ \alpha$. Again $\operatorname{deg}(f)=\operatorname{deg}(\varphi) \operatorname{deg}\left(f_{B}\right)$ and we can conclude as above.

Analogous considerations hold for a hyperelliptic surface, in this case the Albanese variety is an elliptic curve and the fibres of the Albanese map are isomorphic elliptic curves.

\section{The case $\kappa(X)=-\infty$ : toric surfaces}

In this section we are going to show that for a toric surface the presence of curves of negative self-intersection implies that, up to a finite set, degrees of nontrivial self-maps are not square free. Before dealing with the toric case, we briefly recall in general how a nontrivial self-map acts on curves.

Remark 9 Let $X$ be a surface with a nontrivial self-map, $f: X \rightarrow X$. Let $C$ and $D$ be irreducible curves on $X$ such that $f(C)=D$. Since $f_{*} \circ f^{*}=\operatorname{deg}(f) \operatorname{Id}$ on NS(X), there are positive integers $a, b$ such that $f_{*}(C) \equiv_{\text {num }} a D$ and $f^{*}(D) \equiv_{\text {num }} b C$ where $\operatorname{deg} f=a b$. As a consequence, $C^{2}=0$ if and only if $D^{2}=0$, hence the image under a nontrivial self-map of an irreducible curve with zero self-intersection is always a curve with the same property. Analogously $C^{2}<0$ if and only if $D^{2}<0$ and in this case we also have $f^{-1}(D)=C$ : in fact any two distinct components of $f^{-1}(D)$ should be curves with negative self-intersection whose classes in $\mathrm{NS}(X)$ are linearly dependent (by injectivity of $f_{*}$, see [8, §2] for instance). Hence, if we denote

$$
\mathcal{S}_{X}=\left\{C \text { irreducible curve } \mid C^{2}<0\right\}
$$

then the map of sets $\hat{f}: \mathcal{S}_{X} \rightarrow \mathcal{S}_{X}, C \mapsto f(C)$, is bijective.

The proof of Proposition 11 below will make use of the following elementary statement. Since we will need it in subsequent sections we state it in the form of a lemma.

Lemma 10 Let $X$ be a surface that contains one and only one curve $C$ such that $C^{2}<0$. If $f: X \rightarrow X$ is a nontrivial self-map then $\operatorname{deg}(f)$ is a square.

Proof In view of the above Remark 9 we have $f(C)=C, f^{*} C \equiv_{\text {num }} a_{1} C$ and $f_{*} C \equiv_{\text {num }} a_{2} C$ for suitable integers $a_{1}, a_{2}$ such that $\operatorname{deg} f=a_{1} a_{2}$. By the Projection Formula

$$
a_{1} C^{2}=f^{*} C \cdot C=C \cdot f_{*} C=a_{2} C \cdot C=a_{2} C^{2}
$$

hence $a_{1}=a_{2}$ and $\operatorname{deg} f$ is a square.

Proposition 11 The only toric surface admitting self-maps of any given degree is $\mathbb{P}^{1} \times \mathbb{P}^{1}$. If $S \not \mathbb{P}^{1} \times \mathbb{P}^{1}$ is a toric surface, then $S$ fails to have a self-map of prime degree for all but at most a finite number of primes. 
Proof Let $X$ be a toric surface, by the classification of toric surfaces, see [10, Theorem 1.28, p. 42] for example, $X$ is obtained by a finite number of equivariant blow-ups from the projective plane or a Hizerbruch surface $\mathbb{F}_{n}, n \geq 0$. The only cases in which $\mathcal{S}_{X}$ is empty are either $X \simeq \mathbb{P}^{2}$ or $X \simeq \mathbb{P}^{1} \times \mathbb{P}^{1}$. All the nontrivial self-maps of the former have degree a square, the latter instead has nontrivial self-maps of any given degree.

Suppose now that $\mathcal{S}_{X}$ is nonempty. Since $X$ is a toric surface, any irreducible curve on $X$ with negative self-intersection is included in the complement of the torus, hence $\mathcal{S}_{X}$ is finite. If $\mathcal{S}_{X}$ consists of only one element, then our claim follows by Lemma 10. If $\mathcal{S}_{X}$ contains more than one element then $X$ is not minimal as follows by the classification of rational surfaces. Let $D \in \mathcal{S}_{X}$ be a -1 -curve on $X$ and $C \in \mathcal{S}_{X}$ such that $f(C)=D$, recall Remark 9. We have

$$
b^{2} C^{2}=f^{*} D \cdot f^{*} D=f^{*}(D \cdot D)=-\operatorname{deg}(f)
$$

and since $\mathcal{S}_{X}$ is finite $C^{2}$ in (3) can only take a finite number of values. It follows that apart from a finite number of values $\operatorname{deg}(f)$ is not square free.

\section{The case $\kappa(X)=-\infty: \mathbb{P}^{1}$-bundles over a non rational curve}

First of all we are going to introduce notations that will be used in this and the next section.

Notations 12 We denote by $\mathcal{E}$ a locally free sheaf of rank two over a curve $B$ of genus $g(B)$ greater than or equal to one, and set $X=\mathbb{P}(\mathcal{E})$. Moreover we denote by $\pi: X \rightarrow B$ the projection associated to the projective bundle structure. Since $g(B) \geq 1$, given a nontrivial self-map $f: X \rightarrow X$, it induces a nontrivial self-map of $B$ that we denote by $f_{B}$.

Note that $\operatorname{deg}(f)=\operatorname{deg}\left(f_{B}\right) \cdot \operatorname{deg}\left(f_{\mathbb{P}^{1}}\right)$, where $\operatorname{deg}\left(f_{\mathbb{P}^{1}}\right)$ denotes the degree of $f$ when restricted to a fiber, this degree does not depend on the particular chosen fiber. Moreover if $\operatorname{deg}\left(f_{B}\right)>1$ i.e. $f_{B}$ is a nontrivial surjective endomorphism, then $B$ is an elliptic curve.

We are going to analyze case (vi) of Theorem 3. The main result of this section is the following:

Theorem 13 Let $\mathcal{E}$ be a rank two vector bundle over a projective curve $B$ of genus $g>1$. The projective bundle $X=\mathbb{P}(\mathcal{E})$ admits a nontrivial self-map of degree $n$ for every positive integer $n$ if and only if

(i) $\mathcal{E} \simeq \mathcal{O} \oplus \mathcal{L}$ where $\mathcal{L}$ is either a trivial or a torsion line bundle of order $p$ a prime and in the latter case,

(ii) for every $m \in(\mathbb{Z} / p \mathbb{Z})^{*}$ there exists an automorphism $\varphi \in \operatorname{Aut}(B)$ such that $\varphi^{*}(\mathcal{L}) \simeq \mathcal{L}^{ \pm m}$

Before proving the above theorem we will recall some well known facts on $\mathbb{P}^{1}$-bundles over a smooth projective curve, and in the meantime we will establish some notations.

Remark 14 Suppose that $X=\mathbb{P}(\mathcal{E})$, where $\mathcal{E}$ is a locally free sheaf of rank two of degree $e$ on the smooth curve $B$. We have $\operatorname{NS}(X)=\mathbb{Z} H+\mathbb{Z} F$, where $H$ is a divisor such that $\mathcal{O}_{X}(H) \simeq \mathcal{O}(1)$ and $F$ is a fiber of the projection $\pi$. If $D$ is a divisor on $X$ such that $D^{2}=0$ then its class in $\mathrm{NS}(X)$ is either a multiple of $F$ or a multiple of $H+\frac{e}{2} F$. 
If $f: X \rightarrow X$ is a nontrivial self-map that induces an automorphism on the base $B$, then the ramification divisor $R$ of $f$ satisfies $R \equiv_{\operatorname{lin}} K_{X / B}-f^{*} K_{X / B}$ and moreover we have $K_{X / B} \equiv_{\text {num }}-2 H-e F$. It follows that $K_{X / B}^{2}=0$ and $\left(f^{*} K_{X / B}\right)^{2}=0$, which implies $f^{*} K_{X / B} \equiv_{\text {num }}-2(\operatorname{deg} f) H-(\operatorname{deg} f) e F$. Summing up we have

$$
R \equiv_{\text {num }}(1-\operatorname{deg} f) K_{X / B} \text { and } \quad R^{2}=0 .
$$

Remark 15 If $X=\mathbb{P}(\mathcal{E})$ admits two disjoint sections, then $\mathcal{E}$ is isomorphic to the direct sum of two line bundles, and $X=\mathbb{P}(\mathcal{O} \oplus \mathcal{L})$ up to an isomorphism obtained tensoring by a suitable line bundle. If $\mathcal{L}$ is nontrivial, then we denote by $S_{1}, S_{2}$ the sections of $X$ corresponding respectively to the line bundles $\mathcal{O}$ and $\mathcal{L}$, and by $s_{1}, s_{2}: B \rightarrow X$ the associated embeddings. We have $\mathcal{N}_{S_{1} / X} \simeq \mathcal{N}_{S_{2} / X}^{\vee} \simeq \mathcal{L}, S_{1}^{2}=\operatorname{deg}(\mathcal{L})=e$ and $S_{2}^{2}=-\operatorname{deg}(\mathcal{L})=-e$.

If $\operatorname{deg}(\mathcal{L})>0$, then $S_{2}$ is the only curve on $X$ of negative self-intersection. In case $\operatorname{deg}(\mathcal{L})=0$ and $\mathcal{L}$ is nontrivial, the curves $S_{1}, S_{2}$ are the only sections of zero selfintersection and any class in $\mathrm{NS}(X)$ whose square equals zero is either a multiple of the class of $H$ or a multiple of the class of $F$.

Remark 16 Curves dominating $B$ and of zero self-intersection on $X=\mathbb{P}(\mathcal{O} \oplus \mathcal{L}), \mathcal{L}$ a torsion line bundle of order $k$, will play a central role in the proof of Theorem 13. A particular curve of this type is given by the étale cyclic cover $j: \widetilde{B} \rightarrow B$ of degree $k$ determined by $\mathcal{L}$. We are going to recall how to construct $\widetilde{B}$, see for instance [1, p. 54]. Denote by $\mathbf{L}$ the total space of $\mathcal{L}$, and by $\operatorname{pr}_{\mathbf{L}}: \mathbf{L} \rightarrow B$ the bundle projection. The zero divisor of the section $1-l^{k}$ in $\mathbf{L}$, where $l \in \Gamma\left(\mathbf{L}, \operatorname{pr}_{\mathbf{L}}^{*} \mathcal{L}\right)$ is the tautological section, is the curve $\widetilde{B}$ and $j$ is the restriction to $\widetilde{B}$ of the bundle map. Observe that there is a canonical isomorphism of varieties $\mathbf{L} \simeq X \backslash S_{2}$, and that through this isomorphism the image of $\widetilde{B}$ is disjoint from $S_{1}$. By the above description the projective irreducible curve $\widetilde{B}$ is a principal divisor in $X \backslash S_{2}$ and the normal bundle to $\widetilde{B}$ in $X$ is trivial.

Let $D \neq S_{1}, S_{2}$ be an irreducible curve in $X$ dominating $B$ such that $D^{2}=0$, we are going to show that $D \subset X \backslash S_{2} \simeq \mathbf{L}$ and there exists $a \in \mathbb{C}^{*}$ such that the automorphism of varieties

$$
\mu_{a}: \mathbf{L} \rightarrow \mathbf{L}
$$

induced by multiplication by $a$, sends $D$ to $\widetilde{B}$. Since $D^{2}=0$ and $D$ dominates $B$, the divisor $D$ is numerically equivalent to a multiple of $S_{1}$ and $S_{2}$, in particular it is disjoint from $S_{1} \cup S_{2}$ and thus lies in the complement of the zero section of $X \backslash S_{2} \simeq \mathbf{L}$ that coincides with $S_{1}$. Since $\widetilde{B}$ is a principal divisor in $X \backslash S_{2}$, the intersection of $\widetilde{B}$ with a different projective curve included in $X \backslash S_{2}$ is empty. Since multiplication by scalars acts transitively on the nonzero elements of the fibres of $\mathbf{L}$, there exists $a \in \mathbb{C}^{*}$ such that $\widetilde{B} \cap \mu_{a}(D)$ is nonempty and this forces $\widetilde{B}=\mu_{a}(D)$. In particular the restriction of $\pi$ to such a $D$ gives an étale covering of $B$ isomorphic to $j: \widetilde{B} \rightarrow B$, and $D \cdot F=k$.

Theorem 13 will be a consequence of the next two propositions. In Proposition 17 we study nontrivial self-maps of surfaces of the form $\mathbb{P}(\mathcal{O} \oplus \mathcal{L})$ where $\mathcal{L}$ is a torsion line bundle on a curve $B$ of genus $g(B) \geq 1$. In Proposition 18 we characterize $\mathbb{P}^{1}$-bundles on a curve of genus greater than one admitting an endomorphism of degree two.

Proposition 17 Suppose $X=\mathbb{P}(\mathcal{O} \oplus \mathcal{L})$, where $\mathcal{L}$ is a torsion line bundle on $B$ of order $k>1$, and the genus $g(B) \geq 1$. $X$ admits a nontrivial self-map $f: X \rightarrow X$ such that $f_{B} \in$ $\operatorname{Aut}(B)$ and $\operatorname{deg}(f)=d$ if and only if either 
(i) $k \mid d$, or

(ii) there exists $\varphi \in \operatorname{Aut}(B)$ such that $\varphi^{*} \mathcal{L} \simeq \mathcal{L}^{m}$ with $d \equiv \pm m \bmod k$.

In case (ii) there exists a nontrivial self-map $f$ of degree $d$ such that $f_{B}=\varphi$ and either $f^{*} S_{i}=d S_{i}$ for $i=1,2$ or $f^{*} S_{i}=d S_{j}$ for $i \neq j$.

Proof We begin by proving the only if part of our statement. Since $f_{B}$ is an automorphism $f\left(S_{i}\right)$ is a section. Moreover, see Remark $9,\left(f_{*}\left(S_{i}\right)\right)^{2}=0$, therefore $f\left(S_{i}\right)=S_{j}$ for $i, j \in\{1,2\}$. Then there are three possible cases

$$
\begin{aligned}
& f\left(S_{1}\right)=S_{1} \quad \text { and } \quad f\left(S_{2}\right)=S_{2} \\
& f\left(S_{1}\right)=S_{2} \quad \text { and } \quad f\left(S_{2}\right)=S_{1} \\
& f\left(S_{1}\right)=f\left(S_{2}\right)=S_{i} \quad \text { for either } i=1 \text { or } i=2
\end{aligned}
$$

and in any of the cases above the pullback of the divisor $S_{j}$ is given by

$$
f^{*} S_{j}=n_{1, j} S_{1}+n_{2, j} S_{2}+\sum_{\iota} k_{\iota} C_{\iota}
$$

where $n_{1, j}, n_{2, j}$ are nonnegative integers, $k_{\iota}$ are positive integers, and $C_{\iota} \neq S_{1}, S_{2}$ are distinct irreducible curves. Since $X$ contains no curve with negative self-intersection, the irreducible components of $f^{*}\left(S_{j}\right)$ are disjoint, $C_{\iota}$ dominates $B$, and $C_{\iota}^{2}=0$ for every $\iota$. By Remark 16 we also know that $C_{\iota}$ intersects transversally every fiber of $\pi$ in $k$ points. Since $f^{*}(F) \equiv_{\text {num }} F$ we are able to recover the degree of $f$ as the intersection number between $F$ and $f^{*} S_{j}$ hence

$$
d=n_{1, j}+n_{2, j}+k \sum_{\iota} k_{\iota}
$$

In case (6) there exists $j$ such that $n_{1, j}=n_{2, j}=0$ and the intersection number between $f^{*} S_{j}$ and $F$ is $k \sum_{\iota} k_{\iota}$. In cases (4)-(5) we have $f\left(S_{1}\right)=S_{j}$ the multiplicity $n_{2, j}$ is zero and $d \equiv n_{1, j} \bmod k, j=1,2$. We have then

$$
f_{B}^{*} \mathcal{N}_{S_{j} / X} \simeq f_{B}^{*} s_{j}^{*} \mathcal{O}\left(S_{j}\right) \simeq s_{1}^{*} f^{*} \mathcal{O}\left(S_{j}\right) \simeq s_{1}^{*} \mathcal{O}\left(n_{1, j} S_{1}\right) \simeq\left(\mathcal{N}_{S_{1} / X}\right)^{n_{1, j}}
$$

Since $\mathcal{N}_{S_{1} / X} \simeq \mathcal{N}_{S_{2} / X}^{\vee} \simeq \mathcal{L}$ we get $f_{B}^{*} \mathcal{L} \simeq \mathcal{L}^{n_{1,1}}$ in case (4) and $f_{B}^{*} \mathcal{L} \simeq \mathcal{L}^{-n_{1,2}}$ in case (5). Setting $\varphi=f_{B}$ we get $\varphi^{*} \mathcal{L} \simeq \mathcal{L}^{m}$ with $d \equiv m \bmod k$ for $m=n_{1,1}$ in case (4) and $\varphi^{*} \mathcal{L} \simeq \mathcal{L}^{m}$ with $d \equiv-m \bmod k$ for $m=-n_{1,2}$ in case (5).

Now we come to the proof of the if part of our statement. Denote by $\mathbf{O}, \mathbf{L}$ respectively the total spaces of $\mathcal{O}$ and $\mathcal{L}$ and by $\mathbf{O}_{x}$ and $\mathbf{L}_{x}$ fibres of the bundle map over the point $x$. First of all suppose that $k \mid d$. In this case, the isomorphism $i: \mathbf{L}^{\otimes d} \rightarrow \mathbf{O}$ enables us to construct a degree $d$ morphism of varieties

$$
\begin{gathered}
\Phi: \mathbf{O} \oplus \mathbf{L} \rightarrow \mathbf{O} \oplus \mathbf{L}^{\otimes d-1} \\
(\alpha, l) \in \mathbf{O}_{x} \oplus \mathbf{L}_{x} \mapsto\left(\alpha^{d}+i\left(l^{\otimes d}\right), \alpha \cdot l^{\otimes d-1}\right) \in \mathbf{O}_{x} \oplus \mathbf{L}_{x}^{\otimes d-1} .
\end{gathered}
$$

Since $\Phi$ is homogeneous on the fibres it induces a degree $d$ morphism

$$
\hat{\Phi}: \mathbb{P}(\mathcal{O} \oplus \mathcal{L}) \rightarrow \mathbb{P}\left(\mathcal{O} \oplus \mathcal{L}^{d-1}\right)
$$


and composing with the canonical isomorphism $\mathbb{P}\left(\mathcal{O} \oplus \mathcal{L}^{d-1}\right) \simeq \mathbb{P}(\mathcal{L} \oplus \mathcal{O})$ induced by tensorization by $\mathcal{L}$ we get the desired endomorphism.

Suppose now that $d \equiv \pm m \bmod k$ and that there is an automorphism of $B$, say $\varphi$, such that $\varphi^{*} \mathcal{L} \simeq \mathcal{L}^{m}$. Since $\mathcal{L}$ is of $k$-torsion, we have a degree $d$ map

$$
\mathbf{O} \oplus \mathbf{L} \rightarrow \mathbf{O} \oplus \mathbf{L}^{d} \simeq \mathbf{O} \oplus \mathbf{L}^{ \pm m} \simeq \mathbf{O} \oplus \varphi^{*} \mathbf{L}^{ \pm 1}
$$

given on the fibres by

$$
(\alpha, l) \mapsto\left(\alpha^{d}, l^{\otimes d}\right)
$$

and hence a map $\phi_{1}: \mathbb{P}(\mathcal{O} \oplus \mathcal{L}) \rightarrow \mathbb{P}\left(\mathcal{O} \oplus \varphi^{*} \mathcal{L}\right)$ of degree $d$ that induces the identity on the base. Moreover the natural map

$$
\phi_{2}: \mathbb{P}\left(\mathcal{O} \oplus \varphi^{*} \mathcal{L}^{ \pm 1}\right) \simeq \mathbb{P}\left(\mathcal{O} \oplus \mathcal{L}^{ \pm 1}\right) \times_{\varphi} B \rightarrow \mathbb{P}\left(\mathcal{O} \oplus \mathcal{L}^{ \pm 1}\right) \simeq \mathbb{P}(\mathcal{O} \oplus \mathcal{L})
$$

is an isomorphism that induces $\varphi$ on the base. It follows that $\phi=\phi_{1} \circ \phi_{2}$ is a nontrivial selfmap of $\mathbb{P}(\mathcal{O} \oplus \mathcal{L})$ that induces $\varphi$ on the base. The final part of the statement holds setting $f=\phi$.

Proposition 18 Let $X=\mathbb{P}(\mathcal{E}) \rightarrow B$ be a projective bundle, with $\mathcal{E}$ a locally free sheaf of rank two on $B$ a curve of genus $g(B) \geq 2$. Suppose $X$ admits a nontrivial self-map $f$ of degree two, then either

(i) $X=\mathbb{P}(\mathcal{O} \oplus \mathcal{L})$ with $\mathcal{L}$ a torsion line bundle, or

(ii) The ramification divisor $R_{f}$ of $f$ is a smooth irreducible curve, the restriction of $\pi$ to $R_{f}$ is an étale double covering of $B$ and the normal bundle $\mathcal{N}_{R_{f} / X}$ to $R_{f}$ in $X$ is a torsion line bundle of order strictly greater than 2 .

Proof Let $f: X \rightarrow X$ be a nontrivial self-map of degree two. Since $f_{B}$ is an automorphism, the restriction of $f$ to every fiber is a double covering of $\mathbb{P}^{1}$, hence it ramifies at exactly two points. It follows that $R_{f}$ is a smooth curve intersecting transversally every fiber of $\pi$ in two points. Therefore $R_{f}$ is either union of two disjoint sections $S_{1}$ and $S_{2}$ or it is irreducible and an étale double covering of $B$.

In the first case each one of this sections has zero self-intersection by Lemma 10. If $X$ is not the trivial projective bundle then $S_{1}$ and $S_{2}$ are the unique sections of zero selfintersection. Moreover the image $T=f\left(S_{1}\right)$ is a section of zero self-intersection by Remark 9, therefore $T=S_{j}$ for either $j=1$ or $j=2$. As a particular case of (8) we get

$$
f_{B}^{*} \mathcal{N}_{S_{j} / X} \simeq\left(\mathcal{N}_{S_{1} / X}\right)^{2}
$$

and since $\mathcal{N}_{S_{1} / X} \simeq \mathcal{N}_{S_{2} / X}^{\vee}$ we obtain

$$
\text { either } f_{B}^{*}\left(\mathcal{N}_{S_{1} / X}^{\vee}\right) \simeq\left(\mathcal{N}_{S_{1} / X}\right)^{2} \quad \text { or } \quad f_{B}^{*} \mathcal{N}_{S_{1} / X} \simeq\left(\mathcal{N}_{S_{1} / X}\right)^{2} .
$$

In both cases, since $f_{B}$ has finite order in the group of automorphisms of $B, \mathcal{N}_{S_{1} / X}$ (hence also $\mathcal{N}_{S_{2} / X}$ ) is a torsion line bundle.

We turn our attention to case (ii), namely when $R_{f}$ is irreducible. We denote by $i: X \rightarrow X$ the involution associated with $f$. We may assume that $X$ contains no sections with zero self-intersection. Otherwise, denote by $S$ such a section, the curves $S$ and $S^{\prime}:=i(S)$ are numerically equivalent, hence they are disjoint. It follows that $\mathcal{N}_{S / X}=\mathcal{N}_{S^{\prime} / X}^{\vee}$. 
On the other hand, since $i$ descends to the identity on $B$ it induces an isomorphism $\mathcal{N}_{S / X} \simeq \mathcal{N}_{S^{\prime} / X}$. We conclude $X \simeq \mathbb{P}\left(\mathcal{O} \oplus \mathcal{N}_{S / X}\right)$ and $\mathcal{N}_{S / X}^{\otimes 2} \simeq \mathcal{O}$ and we are in case (i).

We may also assume that $f\left(R_{f}\right)=R_{f}$. Otherwise the image $T=f\left(R_{f}\right)$ satisfies, by Remark $9, T^{2}=0$ and since by the above argument we may suppose that $f(T)$ is not a section, we have that $T \neq i(T)$. Since $T$ and $i(T)$ are numerically equivalent they are disjoint too. Hence making a base change by the étale double covering $\pi_{\mid T}: T \rightarrow B$ we get a $\mathbb{P}^{1}$-bundle over $T$ with four disjoint sections (two of them mapping onto $T$ and the others onto $i(T)$ ), so this projective bundle is trivial and $X$ is the quotient of $T \times \mathbb{P}^{1}$ by a $\mathbb{Z} / 2 \mathbb{Z}$-action without fixed points. Such an action is always diagonal (see Remark 19), hence there exist $p_{1}, p_{2} \in \mathbb{P}^{1}$ such that $T \times p_{i}$ is sent to itself by the $\mathbb{Z} / 2 \mathbb{Z}$-action. The image $S_{i}$ of $T \times p_{i}$ in $X$ is a section and the pullback of its normal bundle to $T \times p_{i}$ is trivial. Hence $\mathcal{N}_{S_{i} / X}$ is a torsion line bundle of order two and we are again in case (i) of the proposition.

Finally assuming $f\left(R_{f}\right)=R_{f}$ and denoting by $\hat{f}: R_{f} \rightarrow R_{f}$ the restriction of $f$ we get $\hat{f}^{*} \mathcal{N}_{R_{f} / S} \simeq \mathcal{N}_{R_{f} / S}^{2}$ and since $\hat{f}$ has finite order, the normal bundle $\mathcal{N}_{R_{f} / S}$ is a torsion line bundle. We can exclude that $\mathcal{N}_{R_{f} / S}^{2} \simeq \mathcal{O}$. Indeed this would imply that $\mathcal{N}_{R_{f} / S}=\mathcal{O}$, and after a base change by the restriction of $\pi$ to $R_{f}$ we get a $\mathbb{P}^{1}$-bundle over $R_{f}$ having two sections with trivial normal bundles, so again it is the trivial $\mathbb{P}^{1}$-bundle and $X$ is as in case (i).

We are now in position to prove Theorem 13.

Proof of Theorem 13 Let $X=\mathbb{P}(\mathcal{E})$ be a surface admitting a self-map of degree $n$ for every $n \in \mathbb{N}$. By Proposition 18 either

(i) $X=\mathbb{P}(\mathcal{O} \oplus \mathcal{L})$ with $\mathcal{L}$ a torsion line bundle, or

(ii) there exists a nontrivial self-map of $X$ of degree two such that the restriction of $\pi$ to the ramification divisor $R_{f}$ of $f$ is a nontrivial étale double covering of $B$ and the normal bundle $\mathcal{N}_{R_{f} / X}$ to $R_{f}$ in $X$ is a torsion line bundle of order strictly greater than 2 .

In case (i) we may suppose that $\mathcal{L}$ is not trivial since otherwise our statement is clearly true. Let $k \geq 2$ be the order of $\mathcal{L}$. By Proposition 17, for every non zero $r \in \mathbb{Z} / k \mathbb{Z}$ there exists $\varphi \in \operatorname{Aut}(B)$ such that $\varphi^{*}(\mathcal{L}) \simeq \mathcal{L}^{ \pm r}$. We only need to remark that $k$ must be prime since pulling back by an automorphism of $B$ preserves the order of a torsion line bundle.

In case (ii) let $m$ be the order of $\mathcal{N}_{R_{f} / X}$.

Claim 1 For every curve $C \neq R_{f}$ on $X$ such that $C^{2}=0$, the intersection number $C \cdot F$ is a multiple of $m$.

Proof of Claim 1 The étale double covering $R_{f} \rightarrow B$ induces an étale double covering $h: R_{f} \times_{B} X \rightarrow X$ and $h^{-1}\left(R_{f}\right)$ is the union of two disjoint sections $S_{1}$ and $S_{2}$ whose normal bundles are $\mathcal{N}_{R_{f} / X}$ and $\mathcal{N}_{R_{f} / X}^{\vee}$. Hence $R_{f} \times_{B} X \simeq \mathbb{P}\left(\mathcal{O} \oplus \mathcal{N}_{R_{f} / X}\right)$. Let $C^{\prime}$ be a component of $h^{-1}(C)$, since $\mathbb{P}\left(\mathcal{O} \oplus \mathcal{N}_{R_{f} / X}\right)$ contains no curve with negative self-intersection we have $C^{\prime 2}=0$. Moreover $C^{\prime} \neq S_{1}, S_{2}$ because $C \neq R_{f}$. By Remark $16, C^{\prime}$ is a degree $m$ cover of $R_{f}$ and, since the degree of $h$ is two, the degree of the restriction of $\pi$ to $C=h\left(C^{\prime}\right) \subset X$ is either $m$ or $2 m$. In both cases $m$ divides $C \cdot F$.

We are going to show now that $X$ does not admit a nontrivial self-map of degree $m$. Indeed, let $g: X \rightarrow X$ be such a map. Pulling back the divisor $R_{f}$ we get

$$
g^{*}\left(R_{f}\right)=a R_{f}+\sum b_{i} C_{i}
$$


where $b_{i}>0$ and $C_{i}^{2}=0$ for all $i$. By Remark 9 the curve $g\left(R_{f}\right)$ has self-intersection zero, moreover since $g$ induces an automorphism on the base $B$, we have $g_{*}\left(R_{f}\right) \cdot F=2<m$. We deduce by Claim 1 that $g\left(R_{f}\right)=R_{f}$. Therefore $a$ is strictly positive too. Intersecting both members of (9) with $F$ we get

$$
2 m=2 a+b m
$$

where $b=\sum_{i} b_{i}$. Since $a>0$ either $b=1$ and $2 a=m$ or $b=0$ and $a=m$. In both cases $a \geq 2$ because $m>2$. Let $\hat{g}: R_{f} \rightarrow R_{f}$ be the restriction of $g$ and denote by $i: R_{f} \rightarrow X$ the closed embedding. Then

$$
\hat{g}^{*}\left(\mathcal{N}_{R_{f} / S}\right)=i^{*} g^{*}\left(\mathcal{O}\left(R_{f}\right)\right)=i^{*}\left(\mathcal{O}\left(a R_{f}\right)\right)=\mathcal{N}_{R_{f} / S}^{a} .
$$

This is absurd because $\hat{g}$ is an automorphism, $\mathcal{N}_{R_{f} / S}$ is a torsion line bundle of order $m$ and $\mathcal{N}_{R_{f} / S}^{a}$ is a torsion line bundle of order $m / a<m$. This proves the 'only if' part of the statement, the 'if' part follows directly from Proposition 17.

It is not immediately clear whether or not surfaces satisfying the characterization of Theorem 13 do exist. In order to provide examples of such surfaces, we are going to reformulate Theorem 13 in terms of étale quotients of trivial projective bundles, this is the content of Corollary 20. This will lead us to explicit examples, see Examples 21.

We start with the following remark in which among other things we will fix notations needed later on.

Remark 19 Let $C$ be a smooth projective curve of genus $g(C)>1$. Let $h: \mathbb{P}^{1} \times C \rightarrow \mathbb{P}^{1} \times C$ be a nontrivial self-map, since $g(C)>1$ we have $h=h_{1} \times h_{2}$ where $h_{1}: \mathbb{P}^{1} \rightarrow \mathbb{P}^{1}$ is a nontrivial self-map and $h_{2}: C \rightarrow C$ is an automorphism.

Let $G$ be a cyclic group of automorphisms of $C$ that acts on $\mathbb{P}^{1} \times C$. For every $g \in G$ we denote by $\Phi_{g}: \mathbb{P}^{1} \times C \rightarrow \mathbb{P}^{1} \times C$ the induced automorphism. In particular $\Phi_{g}=\phi_{g} \times g$ where $\phi_{g}$ is an automorphism of $\mathbb{P}^{1}$. It follows that $G$ acts on $\mathbb{P}^{1}$ too and the action on $\mathbb{P}^{1} \times C$ is the induced diagonal action. Moreover if $g \in G$ is nontrivial, then there exists an affine coordinate $z$ on $\mathbb{P}^{1}$ such that $\phi_{g}(z)=\epsilon z$ where $\epsilon$ is a $m$-th root of the unity.

Corollary 20 Let $X$ be a nontrivial $\mathbb{P}^{1}$-bundle over a smooth projective curve $B$ of genus $g(B)>1$. The surface $X$ admits self-maps of any given degree if and only if there exist a prime number $p$, a curve $C$ and a group $G$ of automorphisms of $C$ acting on $\mathbb{P}^{1}$ such that

(i) $G$ is cyclic of order $p$, it acts freely on $C$, faithfully on $\mathbb{P}^{1}$ and $X$ is a Galois étale quotient $X \simeq\left(\mathbb{P}^{1} \times C\right) / G$.

(ii) For every non zero $\delta \in \mathbb{Z} / p \mathbb{Z}$ there exists an automorphism $\varphi \in \operatorname{Aut}(C)$ such that for every $g \in G$ either $\varphi \circ g=g^{\delta} \circ \varphi$ or $\varphi \circ g=g^{-\delta} \circ \varphi$.

Proof Let $X$ be a nontrivial $\mathbb{P}^{1}$-bundle over a curve $B$ admitting nontrivial self-maps of any given degree. We are going to prove that there exists a prime $p$ such that (i) and (ii) hold. By Theorem 13, $X \simeq \mathbb{P}(\mathcal{O} \oplus \mathcal{L})$ where $\mathcal{L}$ is a $p$-torsion line bundle on $B, p$ a suitable prime. Let $j: C \rightarrow B$ be the Galois étale cover of $B$ determined by $\mathcal{L}, G \subset \operatorname{Aut}(C)$ its Galois group, $G$ is cyclic of order $p$. Let $\bar{j}: X \times{ }_{B} C \rightarrow X$ be the induced Galois étale cover. Since $\mathcal{L}$ pulls back to a trivial line bundle on $C$,

$$
X \times{ }_{B} C \simeq \mathbb{P}^{1} \times C
$$


and $X$ is the quotient of $X \times{ }_{B} C$ by a $G$-action. The action of $G$ on $\mathbb{P}^{1} \times C$ is diagonal (see Remark 19), moreover $G$ acts freely on $C$, and faithfully on $\mathbb{P}^{1}$, otherwise $X$ would be isomorphic to $\mathbb{P}^{1} \times B$.

Let $\delta \in(\mathbb{Z} / p \mathbb{Z})^{*}$ be the representative of a prime $d$ and let $f: X \rightarrow X$ be a nontrivial selfmap of degree $d$. We claim that $f$ lifts to a degree $d$ endomorphism $\tilde{f}: \mathbb{P}^{1} \times C \rightarrow \mathbb{P}^{1} \times C$. As a first step we are going to show that the induced automorphism $f_{B}$ lifts to an automorphism $\widetilde{f}_{C}: C \rightarrow C$. By Proposition 17, $f_{B}^{*}(\mathcal{L}) \simeq \mathcal{L}^{ \pm \delta}$, hence $j^{*} \circ f_{B}^{*}(\mathcal{L}) \simeq j^{*}\left(\mathcal{L}^{ \pm \delta}\right) \simeq \mathcal{O}_{C}$ and there exists an induced regular map of algebraic varieties $\gamma: \mathbb{C} \times C \rightarrow \mathbf{L}$, where $\mathbf{L}$ denotes the total space of the line bundle $\mathcal{L}$. Denote moreover by $s: C \rightarrow \mathbb{C} \times C$ a non zero section of $\mathbb{C} \times C$ and by $\operatorname{pr}_{\mathbf{L}}: \mathbf{L} \rightarrow B$ the bundle map.

The image $\gamma \circ s(C)$ is a projective curve in the complement of the zero section in $\mathbf{L}$, by Remark 16 the restriction of $\mathrm{pr}_{\mathbf{L}}$ to such a curve gives an étale covering of $B$ isomorphic to the étale covering $j: C \rightarrow B$. Denote by $\sigma$ an isomorphism between these two étale covering of $B$, we then have

$$
f_{B} \circ j=\operatorname{pr}_{\mathbf{L}} \circ \gamma \circ s=j \circ \sigma \circ \gamma \circ s
$$

hence $f_{B}$ lifts to the automorphism $\widetilde{f}_{C} \in \operatorname{Aut}(C)$ given by $\widetilde{f}_{C}:=\sigma \circ \gamma \circ s$.

Finally we are going to show that the existence of the lift $\widetilde{f}_{C}$ of $f_{B}$ implies the existence of a lift $\tilde{f}: \mathbb{P}^{1} \times C \simeq X \times{ }_{B} C \rightarrow \mathbb{P}^{1} \times C \simeq X \times{ }_{B} C$ of $f$. Indeed, denoting by $\hat{\pi}: X \times{ }_{B} C \rightarrow C$ the projection on the second factor, we have a commutative diagram

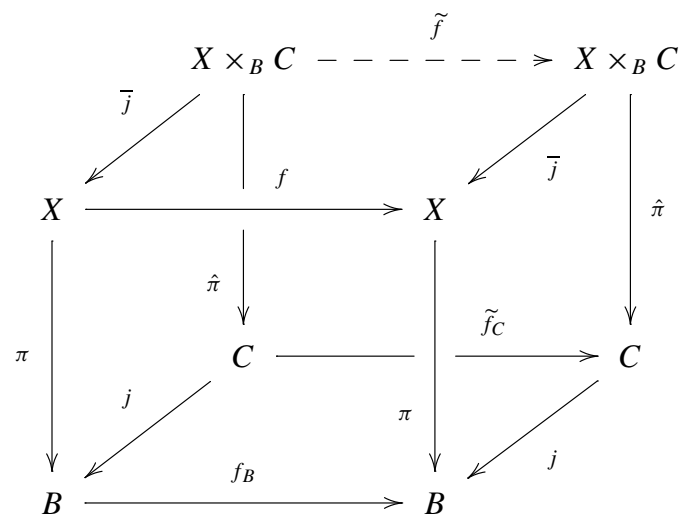

where the dotted arrow $\tilde{f}$ exists by the universal property of the fiber product $X \times{ }_{B} C$ since $\pi \circ f \circ \bar{j}=j \circ \widetilde{f}_{C} \circ \hat{\pi}$. By Remark 19 we have $\widetilde{f}=\psi \times \widetilde{f}_{C}$ where $\psi: \mathbb{P}^{1} \rightarrow \mathbb{P}^{1}$ is a degree $d$ nontrivial self-map of $\mathbb{P}^{1}$. By the last part of Proposition 17, we may also suppose that either $f^{*}\left(S_{i}\right)=d S_{i}$ or $f^{*}\left(S_{i}\right)=d S_{j}$. Since sections $S_{1}$ and $S_{2}$, up to a change of coordinates, lift to $\{0\} \times C$ and $\{\infty\} \times C$ on $\mathbb{P}^{1} \times C$, it follows that the endomorphism $\psi$ is given by either $z \mapsto a z^{d}$ or $z \mapsto b z^{-d}, a, b \in \mathbb{C}^{*}$. Since $\psi \times \widetilde{f}_{C}$ descends to $X$, there exists a positive integer $\delta$ such that for every $g \in G$

$$
\begin{aligned}
\left(\psi \times \tilde{f}_{C}\right) \circ \Phi_{g} & =\left(\psi \times \tilde{f}_{B}\right) \circ\left(\phi_{g} \times g\right) \\
& =\left(\phi_{g}^{\delta} \times g^{\delta}\right) \circ\left(\psi \times \widetilde{f}_{C}\right)=\Phi_{g}^{\delta} \circ\left(\psi \times \widetilde{f}_{C}\right) .
\end{aligned}
$$

Recall that $g \in G$ nontrivial acts on $\mathbb{P}^{1} \times C$ by $\phi_{g}(z, b)=(\epsilon z, g(b))$ where $\epsilon$ is a nontrivial $p$ th root of the unity (Remark 19). The first component of equality (10) gives either $a \epsilon^{d} z^{d}=$ 
$a \epsilon^{\delta} z^{d}$ or $b / \epsilon^{d} z^{d}=b \epsilon^{\delta} / z^{d}$ hence $\delta \equiv \pm d \bmod p$. The second component of equality (10) implies instead $\widetilde{f}_{C} \circ g=g^{ \pm \delta} \circ \widetilde{f}_{C}$ and setting $\varphi:=\widetilde{f}_{C}$ we get item (ii).

To prove the remaining implication observe that the endomorphism of $\mathbb{P}^{1} \times C$ given by $(z, b) \mapsto\left(\frac{1+z^{p}}{z^{p-1}}, b\right)$ always descends to the quotient $X$. The same holds true for the degree $d$ endomorphism, $p \nmid d,(z, b) \mapsto\left(z^{ \pm d}, \varphi(b)\right)$ in case $\varphi \circ g=g^{ \pm \delta} \circ \varphi$.

Examples 21 Fix a prime number $p$, let $A$ be a finite group containing a cyclic group of order $p, \mathbb{Z} / p \mathbb{Z} \subset A$. Let $N_{A}(\mathbb{Z} / p \mathbb{Z})$ be the normalizer of $\mathbb{Z} / p \mathbb{Z}$ in $A$. Letting

$$
\rho_{A}: N_{A}(\mathbb{Z} / p \mathbb{Z}) \rightarrow \operatorname{Aut}(\mathbb{Z} / p \mathbb{Z})
$$

be the homomorphism obtained by conjugation, we denote by

$$
\bar{\rho}_{A}: N_{A}(\mathbb{Z} / p \mathbb{Z}) \oplus \mathbb{Z} / 2 \mathbb{Z} \rightarrow \operatorname{Aut}(\mathbb{Z} / p \mathbb{Z})
$$

the homomorphism given by

$$
(g, \alpha) \mapsto(-1)^{\alpha} \circ \rho_{A}(g) .
$$

Item (ii) of Corollary 20 can be restated asserting the surjectivity of the homomorphism

$$
\bar{\rho}_{\operatorname{Aut}(C)}: N_{\operatorname{Aut}(C)}(\mathbb{Z} / p \mathbb{Z}) \oplus \mathbb{Z} / 2 \mathbb{Z} \rightarrow \operatorname{Aut}(\mathbb{Z} / p \mathbb{Z}) .
$$

To construct examples of surfaces that satisfy the conditions in Theorem 13 or Corollary 20 for any prime $p$, it will be enough to construct a smooth projective curve $C$ of genus $g(C)>1$ such that its automorphisms group contains the group $\mathbb{Z} / p \mathbb{Z}$ as a subgroup acting without fixed points and such that $\bar{\rho}_{\operatorname{Aut}(C)}$ is surjective.

By a classical result of Hurwitz every finite group $\widehat{G}$ can be realized as an automorphism group of a projective curve $C$ of genus greater than or equal to two [5, Corollary 3.15, p. 15]. Moreover we can also realize $\widehat{G}$ as a subgroup of $\operatorname{Aut}(C)$ in such a way that it acts freely on $C$. Indeed such a $\widehat{G}$ fits in a short exact sequence

$$
0 \rightarrow K \rightarrow \Gamma \rightarrow \widehat{G} \rightarrow 0
$$

where $\Gamma$ is a free Fuchsian group that acts freely on the upper half plane $\mathcal{U}$ and $C \simeq \mathcal{U} / K$. But now $C$ is an intermediate covering of the universal covering $\mathcal{U} \rightarrow \mathcal{U} / \Gamma \simeq C / \widehat{G}$ and hence $\widehat{G}$ must act freely.

Finally, choosing $\widehat{G}$ such that $\mathbb{Z} / p \mathbb{Z}$ and $\bar{\rho}_{\widehat{G}}$ is surjective (e.g. taking a semi-direct product $\widehat{G}=\mathbb{Z} / p \mathbb{Z} \ltimes \operatorname{Aut}(\mathbb{Z} / \mathrm{p} \mathbb{Z}))$ and letting $C$ be a curve such that $\widehat{G}$ acts freely on $C$, we get our examples.

\section{$5 \kappa(X)=-\infty: \mathbb{P}^{1}$-bundle over an elliptic curve}

In this section, as the title suggests, we will fix our attention on $\mathbb{P}^{1}$-bundles over an elliptic curve, namely case (v) of Theorem 3. We keep notations introduced at the beginning of the previous section, in particular $\mathcal{E}$ will denote a locally free sheaf of rank two on an elliptic curve $E$.

Remark 22 According to the classification of vector bundles of rank two over an elliptic curve, we may, and will, assume that $X$ is isomorphic to $\mathbb{P}(\mathcal{E})$ where $\mathcal{E}$ is one of the following locally free sheaves: 
(i) $\mathcal{E}=\mathcal{O} \oplus \mathcal{L}$ for a line bundle $\mathcal{L}$ over $E$;

(ii) there is a nontrivial extension

$$
0 \rightarrow \mathcal{O} \rightarrow \mathcal{E} \rightarrow \mathcal{O} \rightarrow 0
$$

(iii) there exists a point $q \in E$ and a nontrivial extension

$$
0 \rightarrow \mathcal{O} \rightarrow \mathcal{E} \rightarrow \mathcal{O}(q) \rightarrow 0
$$

We start by analyzing (ii)-(iii) of Remark 22 .

Proposition 23 Suppose that

$$
0 \rightarrow \mathcal{O} \rightarrow \mathcal{E} \rightarrow \mathcal{O} \rightarrow 0
$$

is a nontrivial extension on the curve $E$. If $f: \mathbb{P}(\mathcal{E}) \rightarrow \mathbb{P}(\mathcal{E})$ is a nontrivial self-map then $f_{E}$ is not an automorphism of E. It follows that there are infinitely many primes that do not appear as degree of a nontrivial self-map of $\mathbb{P}(\mathcal{E})$.

Proof Denote by $S$ the section of $X$ associated to the inclusion of $\mathcal{O}$ in $\mathcal{E}$ given by (11). In order to prove the proposition it is enough to prove that $S$ is the only irreducible curve of zero self-intersection on $X$ that is not contained in a fiber of the projection to $E$. Indeed suppose that $f_{E}$ is an automorphism of $E$, since $R_{f}^{2}=0$ and on $X$ there are no curves of negative self-intersection, the support of $R_{f}$ is union of irreducible curves of zero selfintersection whose projection to $E$ is dominant. If $S$ is the only curve on $X$ of such type, then $R_{f}=m S$ for a suitable integer $m$, but this implies that the restriction of $f$ to the fibres must be a nontrivial self-map of $\mathbb{P}^{1}$ whose ramification divisor is supported on only one point, a contradiction.

Let us prove now that $S$ is the only irreducible curve of zero self-intersection on $X$ that dominates $E$. Suppose that $S^{\prime}$ in another such curve. Since $\left(K_{X / E}+S^{\prime}\right) \cdot S^{\prime}=0, S^{\prime}$ is smooth and $h: S^{\prime} \rightarrow E$ the restriction of the projection $\pi: X \rightarrow E$ to $S^{\prime}$ is étale. After extending the base to $S^{\prime}$ we have

$$
S^{\prime} \times_{E} X=\mathbb{P}\left(h^{*} \mathcal{E}\right),
$$

and $\mathbb{P}\left(h^{*} \mathcal{E}\right)$ has two sections of self-intersection zero, namely the pullback of $S$ and $S^{\prime}$ by the second projection. It follows that $h^{*} \mathcal{E}$ splits, and the pull-back of the extension (11)

$$
0 \rightarrow \mathcal{O}_{S^{\prime}} \rightarrow h^{*} \mathcal{E} \rightarrow \mathcal{O}_{S^{\prime}} \rightarrow 0
$$

is trivial. But the map induced by $h$ on $\operatorname{Ext}^{1}$

$$
\operatorname{Ext}_{E}^{1}(\mathcal{O}, \mathcal{O}) \rightarrow \operatorname{Ext}_{S^{\prime}}^{1}(\mathcal{O}, \mathcal{O})
$$

corresponds in co-homology to

$$
\mathrm{H}^{1}(E, \mathcal{O}) \rightarrow \mathrm{H}^{1}\left(S^{\prime}, \mathcal{O}\right)
$$

which is injective, a contradiction. 
Proposition 24 Let

$$
0 \rightarrow \mathcal{O} \rightarrow \mathcal{E} \rightarrow \mathcal{O}(q) \rightarrow 0
$$

be a nontrivial extension on the elliptic curve $E$. If $f: \mathbb{P}(\mathcal{E}) \rightarrow \mathbb{P}(\mathcal{E})$ is a nontrivial self-map then $\operatorname{deg} f \neq 2$.

Proof Suppose $f$ is a nontrivial self-map of degree two. Let $S$ be the section of $X$ corresponding to the inclusion of $\mathcal{O}$ in $\mathcal{E}$ given by (12) and as usual let $F$ be a fiber of the projection of $\pi: X \rightarrow E$. Since by hypothesis $f$ has degree two and $S^{2}=1$ we have

$$
f^{*} S \cdot f^{*} S=2
$$

Moreover by the Projection Formula

$$
F \cdot f^{*} S= \begin{cases}2 & \text { if } \operatorname{deg}\left(f_{E}\right)=1 \\ 1 & \text { if } \operatorname{deg}\left(f_{E}\right) \neq 1\end{cases}
$$

then

$$
\left(f^{*} S\right)^{2}=\left\{\begin{array}{l}
1+2 b \\
4+4 b
\end{array}\right.
$$

for a suitable integer $b$. In either cases this leads to a contradiction in view of (13).

Now we come to (i) of Remark 22. We will need the following elementary lemma. If $\operatorname{deg}(\mathcal{L})>0$ then $X=\mathbb{P}(\mathcal{O} \oplus \mathcal{L})$ has a unique curve of negative self-intersection and by Lemma 10 every nontrivial self-map of $X$ has degree a square. Hence we may suppose that $\operatorname{deg}(\mathcal{L})=0$.

Lemma 25 Suppose $X=\mathbb{P}(\mathcal{O} \oplus \mathcal{L})$, where $\mathcal{L}$ is a line bundle of degree zero on $E$.

(i) If $\mathcal{L}$ is not a torsion line bundle, then there is no nontrivial self-map of $X$ that induces an automorphism on the curve $E$.

(ii) There exists a nontrivial self-map $f: X \rightarrow X$ that induces isomorphisms on the fibres and a nontrivial self-map $\varphi$ on the base if and only if $\varphi^{*} \mathcal{L}=\mathcal{L}^{ \pm 1}$.

Proof On $X$ there are only two sections of zero self-intersection, we denote them by $S_{1}$ and $S_{2}$, and keep notations introduced in Remark 15. If $C$ is another irreducible curve on $X$ that dominates $E$ such that $C^{2}=0$ and $C \neq S_{1}, S_{2}$, then since the numerical class of $C$ must be a multiple of the numerical class of $S_{1}$ we have $C \cdot S_{i}=0$ and $C$ is disjoint from $S_{1}, S_{2}$. Moreover the restriction of the projection $\pi: \mathbb{P}(\mathcal{O} \oplus \mathcal{L}) \rightarrow E, \pi_{\mid C}: C \rightarrow E$ is étale. Extending the base to $C$ we obtain three sections of zero self-intersection, and then a trivial bundle. But then

$$
\pi_{\mid C}^{*} \mathcal{L}=\left(\pi_{\mid C}^{*} \circ s_{1}^{*}\right) \mathcal{O}\left(S_{1}\right)=\mathcal{O}_{C}\left(S_{1}\right)=\mathcal{O}_{C}
$$

and $\mathcal{L}$ must be a torsion bundle. Suppose then that $S_{1}$ and $S_{2}$ are the only irreducible curves of zero self-intersection on $X$ different from a fiber. Since on $X$ there are no curves of negative self-intersection, if a nontrivial self-map $f$ induces an automorphism on the base 
then the ramification divisor is such that $R_{f}=\operatorname{deg}(f) S_{1}+\operatorname{deg}(f) S_{2}$. We have that $f\left(S_{i}\right)=$ $S_{j}$, and then arguing as in the proof of Proposition 17,

$$
f_{E}^{*} \mathcal{N}_{S_{j} / X}=f_{E}^{*} s_{j}^{*} \mathcal{O}\left(S_{j}\right)=s_{i}^{*} f^{*} \mathcal{O}\left(S_{j}\right)=s_{i}^{*} \mathcal{O}\left(\left(\operatorname{deg}(f) S_{i}\right)=\left(\mathcal{N}_{S_{i} / X}\right)^{\operatorname{deg}(f)} .\right.
$$

Since $\mathcal{N}_{S_{1} / X}=\mathcal{N}_{S_{2} / X}^{\vee}=\mathcal{L}$ the above equalities implies that $f_{E}^{*} \mathcal{L}=\mathcal{L}^{ \pm \operatorname{deg}(f)}$, and then, since $\operatorname{deg}(f) \geq 2, \mathcal{L}$ is a torsion bundle.

We are going to prove item (ii) now. We may suppose that $\mathcal{L}$ is not a trivial sheaf. Since $f$ restricts to an automorphism on the fibres, $f^{*} S_{i} \cdot F=1$ and $f^{-1}\left(S_{i}\right)$ is a section of zero selfintersection, $i=1,2$, hence $f\left(S_{i}\right)=S_{j}$. Again, it follows that either $f_{E}^{*} \mathcal{L}=\mathcal{L}$ or $f_{E}^{*} \mathcal{L}=\mathcal{L}^{\vee}$. This concludes the proof of the only if part of our statement. For the if part, observe that the first projection

$$
X \times_{\varphi} E \rightarrow X
$$

is a finite surjective morphism and since $X \times_{\varphi} E \simeq X$ as projective bundles it induces a nontrivial self-map of $X$ with the desired properties.

Remark 26 As a consequence of Lemma 25 , if $\operatorname{deg}(\mathcal{L})=0$ and $\mathcal{L}$ is not a torsion bundle, then every nontrivial self-map of $\mathbb{P}(\mathcal{O} \oplus \mathcal{L})$ induces an isomorphism on fibres, and the only possible degrees of nontrivial self-maps of $X$ are degrees of nontrivial self-maps of $E$. In view of Corollary 6 we will then assume through the rest of this paper that $X$ is as in case (i) of Remark 22 and $\mathcal{L}$ is a $k$-torsion line bundle.

If $k=1,2,3$, then every prime is either a multiple of $k$ or is congruent to \pm 1 modulo $k$, and by Proposition 17(ii) there exists a nontrivial self-map of any given degree of $X$. If $k>3$ then the existence of nontrivial self-maps of any given degree depends on the geometry of $E$ and $\mathcal{L}$.

Notations 27 We will denote by $E_{i}=\mathbb{C} /(\mathbb{C} \oplus \mathbb{C} i)$ and $E_{\rho}=\mathbb{C} /(\mathbb{C} \oplus \mathbb{C} \rho)$ where $\rho^{3}=1$, $\rho \neq 1$. We have

$$
\operatorname{Aut}\left(E_{i}\right)=\{1,-1, i,-i\} \simeq \mathbb{Z}_{4}
$$

and

$$
\operatorname{Aut}\left(E_{\rho}\right)=\left\{\frac{1}{2}+\frac{\sqrt{3}}{2} i,-\frac{1}{2}+\frac{\sqrt{3}}{2} i,-\frac{1}{2}-\frac{\sqrt{3}}{2} i, \frac{1}{2}-\frac{\sqrt{3}}{2} i,-1,1\right\} \simeq \mathbb{Z}_{6}
$$

moreover if $E \neq E_{i}, E_{\rho}$ then

$$
\operatorname{Aut}(E)=\{1,-1\} \simeq \mathbb{Z}_{2}
$$

where $\operatorname{Aut}(E)$ denotes the automorphism group for the abelian variety structure of an elliptic curve $E$.

Remark 28 We are going to collect in this remark, for reader's convenience, some elementary facts on the ring of endomorphisms of an elliptic curve. Let $E$ be an elliptic curve and $\operatorname{End}(E)$ its ring of endomorphisms. If $E$ does not have complex multiplication then $\operatorname{End}(E)$ is a free $\mathbb{Z}$-module of rank one, every endomorphisms is given by multiplication by $n$, a given integer, and the only possible degrees are squares.

If $E$ has complex multiplication then $\operatorname{End}(E)$ is a rank two free $\mathbb{Z}$-module and $\operatorname{End}_{\mathbb{Q}}(E)=\operatorname{End}(E) \otimes_{\mathbb{Z}} \mathbb{Q}$ is a complex algebraic extension of $\mathbb{Q}$ and $\left[\operatorname{End}_{\mathbb{Q}}(E): \mathbb{Q}\right]=2$. 
Moreover $\operatorname{End}(E) \subseteq \mathcal{O}_{\operatorname{End}_{\mathbb{Q}}(E)}$, i.e. every endomorphism of $E$ is an algebraic integer in $\operatorname{End}_{\mathbb{Q}}(E)$, and

$$
\operatorname{deg}(\alpha)=\mathrm{N}_{\operatorname{End}_{\mathbb{Q}}(E) / \mathbb{Q}}(\alpha)=\alpha \cdot \bar{\alpha}, \quad \forall \alpha \in \operatorname{End}(E),
$$

where $\mathrm{N}_{\text {End }_{\mathbb{Q}}(E) / \mathbb{Q}}()$ denotes the norm of an element in the field extension $\operatorname{End}_{\mathbb{Q}}(E) \mid \mathbb{Q}$ (see $\left[9\right.$, p. 8]). In particular, if a prime number $p$ which is not ramified in $\operatorname{End}_{\mathbb{Q}}(E)$ is the degree of an endomorphism of $E$, then it splits completely in $\operatorname{End}_{\mathbb{Q}}(E)$.

Lemma 29 Suppose $E \neq E_{i}, E_{\rho}$ and $X=\mathbb{P}\left(\mathcal{O}_{E} \oplus \mathcal{L}\right)$ with $\mathcal{L}$ of $k$-torsion. If $X$ has a nontrivial self-map of any given degree then $\varphi(k)<4$, where $\varphi$ denotes the Euler's totient function.

Proof Since we suppose $\operatorname{Aut}(E) \cong \mathbb{Z}_{2}$, if $f: X \rightarrow X$ is a nontrivial self-map of prime degree $p=\operatorname{deg}(f)$ and $f_{E} \in \operatorname{Aut}(E)$ then by Proposition 17 either $p$ is congruent to \pm 1 modulo $k$ or $p=k$. Denote by $\mathfrak{P}_{1}$ the set of primes obtained in the above way, and denote by $\mathfrak{P}_{2}$ the set of primes $p$ such that there exists a nontrivial self-map $f: X \rightarrow X$ with $\operatorname{deg}\left(f_{E}\right)=p$. By Dirichlet Theorem the analytic density of $\mathfrak{P}_{1}$ is less than or equal to $2 / \varphi(k) \leq 1 / 2$. In what follows we may suppose that $E$ has complex multiplication, otherwise the set $\mathfrak{P}_{1} \cup \mathfrak{P}_{2}$ would have analytic density less than or equal to $1 / 2$ and our claim would be clearly true. The set $\mathfrak{P}_{2}$ is then contained, up to a finite set containing ramified primes (recall Remark 28 above), in the set of primes that split completely in the complex quadratic number field $\operatorname{End}_{\mathbb{Q}}(E)$, denote this set by $\mathfrak{P}_{2}^{\prime}$. It follows by Čebotarev density theorem that the analytic density of $\mathfrak{P}_{2}^{\prime}$ is less than or equal to $1 / 2$. If moreover $\varphi(k)>4$ then the analytic density of the set $\mathfrak{P}_{1}$ is strictly less than $2 / 5$ and it follows that $\left(\mathfrak{P}_{1} \cup \mathfrak{P}_{2}\right)^{c}$ contains an infinite number of primes.

Suppose then that $\varphi(k)=4$ and that $\mathfrak{P}_{1} \cup \mathfrak{P}_{2}$ equals the set of primes, then $\mathfrak{P}_{1} \cup \mathfrak{P}_{2}^{\prime}$ has analytic density one. We are going to show that $\mathfrak{P}_{1} \cap \mathfrak{P}_{2}^{\prime}$ has non zero analytic density, and this will lead us to a contradiction. We may suppose that the lattice of $E$ is contained in $\mathbb{Q}(i \sqrt{a})$ where $a$ is a positive square free integer. Observe that

$$
\mathfrak{P}_{2}^{\prime}=\left\{p \text { prime } \mid\left(\frac{-a}{p}\right)=1\right\}
$$

where $(a / p)$ denotes the Legendre symbol, see [11, p. 199]. Write $a=p_{1} \ldots p_{n}$ for the prime decomposition of $a$. Since the Legendre symbol is multiplicative, if $\left(\frac{-1}{p}\right)=1$ and $\left(\frac{p_{i}}{p}\right)=1$ for $i=1 \ldots n$ then $\left(\frac{a}{p}\right)=1$. If $p \equiv 1 \bmod 4 p_{i}$ then $\left(\frac{p_{i}}{p}\right)=1$, indeed for $p_{i}$ odd,

$$
\left(\frac{p_{i}}{p}\right)=(-1)^{\frac{p-1}{2} \frac{p_{i}-1}{2}}\left(\frac{p}{p_{i}}\right)=\left(\frac{p}{p_{i}}\right)
$$

by Gauss' Quadratic Reciprocity Law and

$$
\left(\frac{p}{p_{i}}\right) \equiv p^{\frac{p_{i}-1}{2}} \equiv 1 \bmod p_{i}
$$

by Euler's Criterion. Moreover $\left(\frac{-1}{2}\right)=\left(\frac{2}{p}\right)=1$ if $p \equiv 1 \bmod 8\left[11\right.$, p. 65] for $p_{i}=2$. It follows that $p \in \mathfrak{P}_{1} \cap \mathfrak{P}_{2}^{\prime}$ if $p \equiv 1 \bmod 8 a k$ and the analytic density of the set of primes satisfying this congruence equals $1 / \varphi(8 a k)$. The proof of the Lemma is now complete. 
Remark 30 If $\mathcal{L}$ is a $k$-torsion line bundle, $k=4,6$, then by Proposition 17 the surface $\mathbb{P}(\mathcal{O} \oplus \mathcal{L}), E \neq E_{i}, E_{\rho}$, has nontrivial self-maps of any given prime degree $p \neq 2$ if $k=4$ and $p \neq 2,3$ if $k=6$. Moreover, if $k=4$ then a nontrivial surjective endomorphism $f$ : $X \rightarrow X$ with $\operatorname{deg}(f)=2$, is such that $\operatorname{deg}\left(f_{E}\right)=2$. Analogously if $k=6$ and $\operatorname{deg}(f)=2,3$ then we must have $\operatorname{deg}\left(f_{E}\right)=2,3$ respectively. In general if $k \geq 4$ and $f: X \rightarrow X$ is a nontrivial self-map of degree a prime $p<k$ then we must have $\operatorname{deg}\left(f_{E}\right)=p$.

We are now in position to conclude the analysis of case (i) Remark 22. We will make use of the following elementary Lemma regarding complex multiplications. In what follows we will always suppose that the lattice relative to a given elliptic curve is generated by $1, \tau$, where $\tau$ is a complex number. This does not involve any loss of generality.

Lemma 31 The only possible complex multiplications of degree two are $\pm 1 \pm i, \pm \frac{1}{2} \pm \frac{\sqrt{7}}{2} i$, $\pm \sqrt{2} i$. If an elliptic curve has complex multiplication by $\pm 1 \pm i$ then it is isomorphic to $E_{i}$. If an elliptic curve has complex multiplication by $\pm \frac{1}{2}+ \pm \frac{\sqrt{7}}{2} i$ then it is isomorphic to the curve relative to the lattice $\left\langle 1, \frac{1}{2}+\frac{\sqrt{7}}{2} i\right\rangle$.

Proof For the first part of our statement, recall that if $E$ is an elliptic curve with complex multiplication then $\operatorname{End}_{\mathbb{Q}}(E)=\mathbb{Q}(i \sqrt{a}), a>0$ a rational integer, and $\operatorname{End}(E)$ is contained in the ring of integers of $\mathbb{Q}(i \sqrt{a})$. A direct computation shows then the only complex multiplications of degree two are the ones listed in our statement. If an elliptic curve $E$ has complex multiplication by either $\pm 1 \pm i$ or $\pm \frac{1}{2}+ \pm \frac{\sqrt{7}}{2} i$ then $\operatorname{End}_{\mathbb{Q}}(E)$ coincides respectively with the ring of integers of $\mathbb{Q}(i), \mathbb{Q}(i \sqrt{7})$, say $R$. If $\Lambda$ denotes the lattice of $E$ then $R$. $\Lambda \subseteq \Lambda$ and $\Lambda$ is fractional ideal of $\mathbb{Q}(i), \mathbb{Q}(i \sqrt{7})$ respectively. These two fields have class number one [9, p. 37], so that, after an isomorphism $\Lambda$ becomes equal to $\langle 1, i\rangle,\left\langle 1, \frac{1}{2}+\frac{\sqrt{7}}{2} i\right\rangle$ respectively.

Lemma 32 If $\mathcal{L}$ is a $k$-torsion line bundle on the elliptic curve $E$, with $k \geq 4$, then the surface $X=\mathbb{P}(\mathcal{O} \oplus \mathcal{L})$ admits nontrivial self-maps of any given degree if and only if

- $k=4, E$ is the elliptic curve relative to the lattice $\left\langle 1, \frac{1}{2}+\frac{i \sqrt{7}}{2}\right\rangle$, and $\mu^{*} \mathcal{L}=0$ where $\mu$ is the endomorphism of $E$ induced by multiplication by either $\frac{3}{2}+i \frac{\sqrt{7}}{2}$ or $\frac{3}{2}-i \frac{\sqrt{7}}{2}$.

- $k=5, E=E_{i}$ and $\mu^{*} \mathcal{L}=0$ where $\mu$ is the endomorphism of $E$ induced by multiplication by either $2+i$ or $2-i$.

- $k=7, E=E_{\rho}$ and $\mu^{*} \mathcal{L}=0$ where $\mu$ is the endomorphism of $E$ induced by multiplication by either $\frac{5}{2}+\frac{i \sqrt{3}}{2}$ or $\frac{5}{2}-\frac{i \sqrt{3}}{2}$.

Proof Suppose for the moment that $E \neq E_{i}, E_{\rho}$. As already observed, see Remark 30, $X$ admits a nontrivial self-map of degree two if and only if we can lift a nontrivial self-map, say $\alpha$, of degree two of $E$. Thanks to Lemma 25 this is possible if and only if $\alpha^{*} \mathcal{L}=\mathcal{L}$ or what is equivalent

$$
(1-\alpha)^{*} \mathcal{L}=0 .
$$


By Lemma 31, $\alpha= \pm \frac{1}{2} \pm \frac{\sqrt{7}}{2} i, \pm \sqrt{2} i$. In the latter case $(1-\alpha)$ has degree three ${ }^{1}$ and (15) implies either $k=3$ or $k=1$ a contradiction. If $\alpha=\frac{1}{2} \pm \frac{\sqrt{7}}{2} i$ then (15) implies $k=2$ a contradiction. If $\alpha=-\frac{1}{2} \pm \frac{\sqrt{7}}{2} i$ then (15) implies $k=4, \mathcal{L}$ is in the kernel of either $\frac{3}{2}+i \frac{\sqrt{7}}{2}$ or $\frac{3}{2}-i \frac{\sqrt{7}}{2}$. In either cases we are able to lift $\alpha$ and $X$ admits a nontrivial self-map of any given degree, see Remark 30.

Suppose now that $E=E_{i}$. In order for $X$ to admit a nontrivial self-map of degree two either we are able to lift $\pm 1 \pm i$ or $i^{*} \mathcal{L}=\mathcal{L}^{ \pm 2}$. Hence $k=5, \mathcal{L}$ is in the kernel of either $2+i$ or $2-i$ and by Proposition 17 we have nontrivial self-maps of any given degree.

Finally let $E=E_{\rho}$. In this case the only way to obtain a nontrivial self-map of degree two is to lift $\psi \in \operatorname{Aut}(E)$ with $\psi^{*} \mathcal{L}=\mathcal{L}^{ \pm 2}$. But this last condition implies that $k=7$ and $\mathcal{L}$ is in the kernel of either $\frac{5}{2}+\frac{i \sqrt{3}}{2}$ or $\frac{5}{2}-\frac{i \sqrt{3}}{2}$. It follows that there is an element $\lambda \in \operatorname{Aut}\left(E_{\rho}\right)$ such that $\lambda^{*} \mathcal{L}=\mathcal{L}^{2}$ hence and $(\lambda)^{*}(\lambda)^{*} \mathcal{L}=\mathcal{L}^{4}$. By Proposition 17 we have nontrivial self-maps of any given degree.

Finally, for reader's convenience, we collect results obtained in this section in the following Corollary. It provides the last step in establishing Theorem 2.

Corollary $33 X$ is $\mathbb{P}^{1}$-bundle over an elliptic curve $E, \mathbb{P}(\mathcal{O} \oplus \mathcal{L})$, where $\mathcal{L}$ is a $k$-torsion line bundle on $E$ and either $k=1,2,3$ or

- $k=4, E$ is the elliptic curve relative to the lattice $\left\langle 1, \frac{1}{2}+\frac{i \sqrt{7}}{2}\right\rangle$, and $\mu^{*} \mathcal{L}=0$ where $\mu$ is the endomorphism of $E$ induced by multiplication by either $\frac{3}{2}+i \frac{\sqrt{7}}{2}$ or $\frac{3}{2}-i \frac{\sqrt{7}}{2}$.

- $k=5, E=E_{i}$ and $\mu^{*} \mathcal{L}=0$ where $\mu$ is the endomorphism of $E$ induced by multiplication by either $2+i$ or $2-i$.

- $k=7, E=E_{\rho}$ and $\mu^{*} \mathcal{L}=0$ where $\mu$ is the endomorphism of $E$ induced by multiplication by either $\frac{5}{2}+\frac{i \sqrt{3}}{2}$ or $\frac{5}{2}-\frac{i \sqrt{3}}{2}$.

Proof This is an immediate consequence of Remark 26, Proposition 23, Proposition 24 and Lemma 32.

Acknowledgements The authors would like to thank Massimiliano Mella who suggested this problem to the second author many years ago.

\section{References}

1. Barth, W.P., Hulek, K., Peters, C.A.M., Van de Ven, A.: Compact Complex Surfaces, 2nd edn. Ergebnisse der Mathematik und ihrer Grenzgebiete. 3. Folge. A Series of Modern Surveys in Mathematics, vol. 4. Springer, Berlin (2004) [Results in Mathematics and Related Areas. 3rd Series. A Series of Modern Surveys in Mathematics]

2. Beauville, A.: Complex Algebraic Surfaces, 2nd edn. London Mathematical Society Student Texts, vol. 34. Cambridge University Press, Cambridge (1996). Translated from the 1978 French original by R. Barlow, with assistance from N.I. Shepherd-Barron and M. Reid

3. Beauville, A.: Endomorphisms of hypersurfaces and other manifolds. Int. Math. Res. Not. 1, 53-58 (2001)

4. Birkenhake, C., Lange, H.: Complex Abelian Varieties, 2nd edn. Grundlehren der Mathematischen Wissenschaften, vol. 302. Springer, Berlin (2004) [Fundamental Principles of Mathematical Sciences]

\footnotetext{
${ }^{1}$ Under the identification between points of $E$ and line bundles of degree zero modulo linear equivalence $\alpha^{*}$ operates on points of $E$ as $\hat{\alpha}$ the dual endomorphism of $\alpha$, i.e. $\alpha \circ \hat{\alpha}=\hat{\alpha} \circ \alpha=[\operatorname{deg}(\alpha)]_{E} \cdot \hat{\alpha}$ is obtained from $\alpha$ by complex conjugation.
} 
5. Breuer, T.: Characters and Automorphism Groups of Compact Riemann Surfaces. London Mathematical Society Lecture Note Series, vol. 280. Cambridge University Press, Cambridge (2000)

6. Fujimoto, Y.: Endomorphisms of smooth projective 3-folds with non-negative Kodaira dimension. Publ. Res. Inst. Math. Sci. 38(1), 33-92 (2002)

7. Lang, S.: Algebra, 3rd edn. Graduate Texts in Mathematics, vol. 211. Springer, New York (2002)

8. Nakayama, N.: Ruled surfaces with non-trivial surjective endomorphisms. Kyushu J. Math. 56(2), 433446 (2002)

9. Neukirch, J.: Algebraic Number Theory. Grundlehren der Mathematischen Wissenschaften, vol. 322. Springer, Berlin (1999) [Fundamental Principles of Mathematical Sciences. Translated from the 1992 German original and with a note by Norbert Schappacher, with a foreword by G. Harder]

10. Oda, T.: Convex bodies and algebraic geometry. Ergebnisse der Mathematik und ihrer Grenzgebiete (3), vol. 15. Springer, Berlin (1988) [Results in Mathematics and Related Areas (3). An Introduction to the Theory of Toric Varieties, translated from the Japanese]

11. Ribenboim, P.: Classical Theory of Algebraic Numbers. Springer, New York (2001). Universitext 